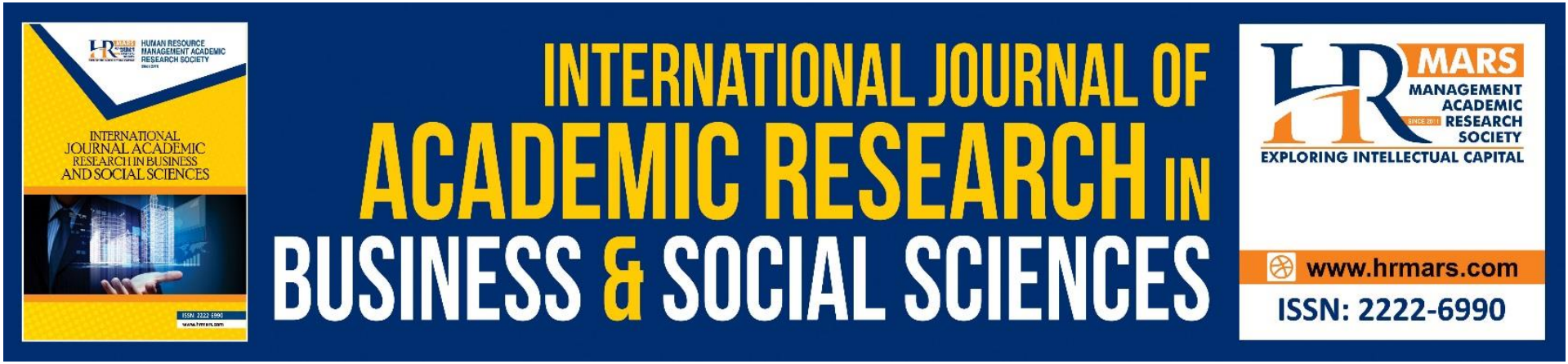

\title{
An Overview of Pandemic Covid-19 and The Repercussion to The Tourism Industries
}

\section{Quratul Aini Binti Roshisham, Siti Yuliandi Binti Ahmad}

To Link this Article: http://dx.doi.org/10.6007/IJARBSS/v11-i10/11449

DOI:10.6007/IJARBSS/v11-i10/11449

Received: 07 August 2021, Revised: 12 September 2021, Accepted: 02 October 2021

Published Online: 19 October 2021

In-Text Citation: (Roshisham \& Ahmad, 2021)

To Cite this Article: Roshisham, Q. A. B., \& Ahmad, S. Y. B. (2021). An Overview of Pandemic Covid-19 and The Repercussion to The Tourism Industries. International Journal of Academic Research in Business and Social Sciences, 11(10), 780-798.

\section{Copyright: (C) 2021 The Author(s)}

Published by Human Resource Management Academic Research Society (www.hrmars.com)

This article is published under the Creative Commons Attribution (CC BY 4.0) license. Anyone may reproduce, distribute, translate and create derivative works of this article (for both commercial and non-commercial purposes), subject to full attribution to the original publication and authors. The full terms of this license may be seen at: http://creativecommons.org/licences/by/4.0/legalcode

\section{Vol. 11, No. 10, 2021, Pg. $780-798$}

Full Terms \& Conditions of access and use can be found at http://hrmars.com/index.php/pages/detail/publication-ethics 


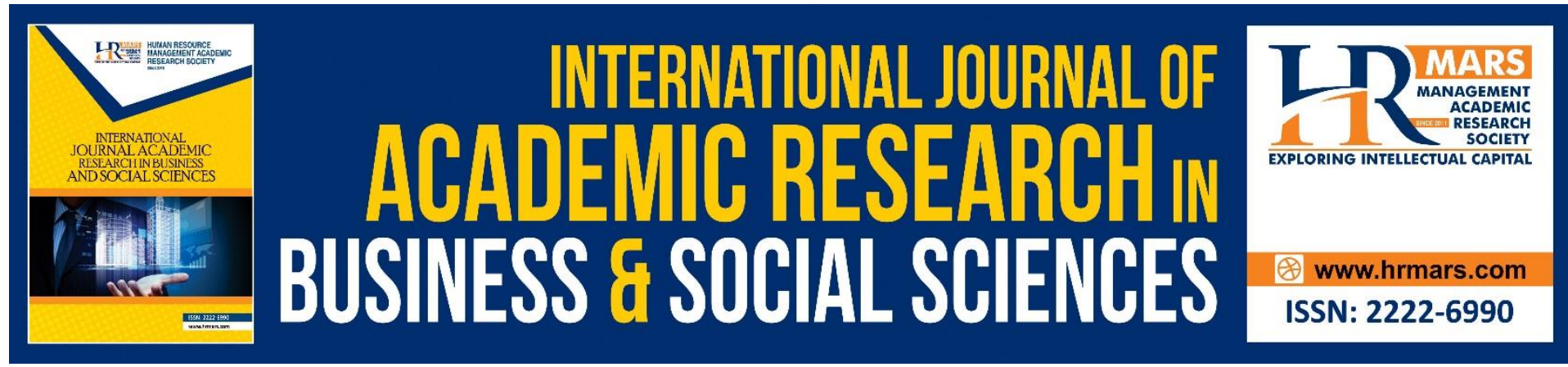

\title{
An Overview of Pandemic Covid-19 and The Repercussion to The Tourism Industries
}

\author{
Quratul Aini Binti Roshisham, Siti Yuliandi Binti Ahmad \\ International Islamic University Malaysia (IIUM) \\ Email: quratul1011@gmail.com, sitiyuliandi@iium.edu.my
}

\begin{abstract}
Novel Coronavirus or COVID-19 is a global pandemic affecting most businesses and sectors worldwide since the first quarter of 2020. Many tour operators and workers are unemployed and leave unpaid as far as tourism is concerned. Moreover, the airline industry is a significant business that suffers severe global losses in the tourist industry. This study synthesises the current research on the tourist impact of the COVID-19 pandemic via the systemic evaluation of ten influential journals and research publications. The findings show that the tourist business of the nation was indirectly damaged, as a result of the pandemic growth, by most of the non-operated or partially shuttered sectors and industries, such as accommodation, transportation, and airlines. The report shows the worldwide tourism industry with severe repercussions since the COVID-19 pandemic halted airlines and touristic activities worldwide. This paper discusses how other countries adaptation strategies may be worth adapting by Malaysia to overcome challenges and barriers. For example, each country can overcome the problem in its approach, and one method is to help the poor financially. The Government may assist local businesses by encouraging domestic shopping to boost the economy and recommend that people support the local products and services since this would indirectly increase the country's economic growth. It also helps small businesses to address the changing demands of tourist companies and new needs not simply by cutting their taxes or through targeted investment in sustainable tourism and a sustainable economy, which is led by a new strategy. Moreover, tour operators are also aware of the need to restructure and anticipate government financial aid to solve the issue and concentrate more on shortening, persevering, and focusing on short-term rather than long-term planning, such as innovation in the pandemic Covid-19.
\end{abstract}

Keywords: Adaptation Strategies, Overview, COVID-19, Tourism Industry, Tour Operators

\section{Introduction}

The novel coronavirus is known as SARS-CoV-2, namely Covid-19. On 31 December 2019, WHO first discovered this novel virus after a report from the Wuhan Cluster of Viral Pneumonia patients based on WHO (2021). The flow of people in the pandemic is changed, with all the people following the new guideline from WHO. The procedures include wearing facial masks in public spaces and constantly washing hands. This pandemic has transformed the world, and this pandemic has dramatically impacted many people, organisations, sectors, 
and businesses. The tourist sector is one of the sectors affected most during the pandemic. Tourism is one of the essential sectors worldwide as it contributes most of the GDP in each country, according to the World Travel and Tourism Council (2020). Most individuals like to travel with friends and family peacefully, and they did so until the Covid-19 pandemic started back in China in December 2019. Following the propagation of Covid-19 worldwide, most tourism operators temporarily closed their businesses and facilities to curb the pandemic. Some of the Government's attempts to partially or lock down all the country's residents (Han et al, 2020). Below is the graph of the worldwide international tourist arrival during the pandemic Covid-19.

Figure 1 shows that the international tourist arrivals worldwide are decreasing terrifically. The most affected region is in Asia \& The Pacific with $-82 \%$ with the average of the declining number is $-72 \%$. The plummet of the tourism industry during the pandemic Covid-19 indirectly affected the economy of the country. When many sectors have to shut down temporarily or directly, it has impacted the economy and created more unemployment, unpaid leaves, and others. The COVID-19 pandemic has seriously deficient banks, according to Shohini Roy (2020). The globe continues to transmit coronavirus and let borrowers and companies through unemployment, fewer sales and lower profits. Banks help to limit coronaviral development. Many banks encourage employees to work from home. Businesses are establishing digital banks to make internet banking more accessible for customers to restrict the spread of coronaviruses. In addition, COVID-19 outbreaks lowered economic recovery potential due to a broad economic slowdown last year. Below is the world real GDP growth rate graph by Dídac García Mancebo and Yukari Hirota (2020). Based on the figure, the world real GDP growth rate declined terrifically between 2019 until 2020.

\section{World real GDP growth rate}

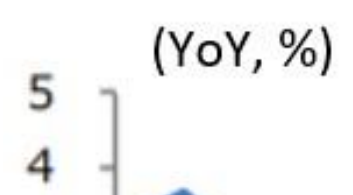




\section{LITERATURE REVIEW}

\section{Figure 2: World GDP growth rates}

Source: Mancebo and Hirota (2020).

This research deliberately sought the impact of Covid-19 on the tourism and economy sector. In this part, there will be a discussion of the literature review regarding the pandemic COVID19 , tourism and economy.

\section{Pandemic Covid-19}

Coronavirus was initially found in Wuhan, China and caused a widespread pandemic. There were first occurrences in China, but the virus spread across Europe and then into the Americas. This virus ravaged Italy, and 233,836 cases of COVID-19 were positive, with 33,601 deaths (Roy, 2020).

The global pandemic of COVID-19 challenged globalisation and international commercial activities, indirectly disturbing the key socioeconomic institutions. A continuous and new start is required for a quick recovery. It returns to economic, employment and trade activities in the financial sectors most impacted (Ruwan Ranasinghe et al., 2020).

\section{Tourism in Covid-19}

Tourism is a significant industry in the global economy. The tourism sector in 2019 accounted for $29 \%$ of the world's service exports and over 300 million jobs. It is an essential source of income and employment for industrialised and developing countries. The worldwide drop in tourist numbers may have disastrous economic repercussions, as many developing nations are mainly dependent on tourism. According to the United Nations Conference on Trade and 
Development (2021), tourism accounts for over half of the GDP of several nations, including numerous small developing island countries (SIDS).

Furthermore, one of the main problems faced by government limitations due to the pandemic is tourism, according to Nagaj and Žuromskaite (2020). The pandemic resulted in local and worldwide tourism movement and travel restrictions.

\section{Methodology}

These authors reviewed secondary data on twenty comprehensive research on the pandemic COVID-19 from 2020 until 2021. Secondary data was synthesised in this overview to deeply understand the current situation of the sectors in the pandemic of Covid-19. The results are illustrated in Table 1 with a summary and discussion to answer the objective of this paper.

Results on Covid19 and Tourism Industry

Table 1: Result on COVID-19 and tourism industry

\begin{tabular}{|c|c|c|c|c|c|}
\hline $\begin{array}{l}\text { AUTHORS } \\
\text { AND } \\
\text { COUNTRY }\end{array}$ & $\begin{array}{l}\text { COMPONENTS } \\
\text { OF RESEARCH } \\
\text { FOCUS }\end{array}$ & $\begin{array}{l}\text { METHODO } \\
\text { LOGY }\end{array}$ & RESULTS & $\begin{array}{l}\text { CONCLUSIO } \\
\mathbf{N}\end{array}$ & REMARKS \\
\hline $\begin{array}{l}\text { - Wasiul } \\
\text { Karim, } \\
\text { Ahasanu } \\
\text { I Haque, } \\
\text { Zohurul } \\
\text { Anis \& } \\
\text { Moham } \\
\text { mad } \\
\text { Arije } \\
\text { Ulfy } \\
\text { (2020) } \\
\text { Malaysi } \\
\text { a }\end{array}$ & $\begin{array}{l}\text { - } \text { Future } \\
\text { impact } \\
\text { - Restricted } \\
\text { movement } \\
\text { control } \\
\text { order for } \\
\text { Covid-19. }\end{array}$ & $\begin{array}{ll} & \text { Case } \\
& \text { Study }\end{array}$ & 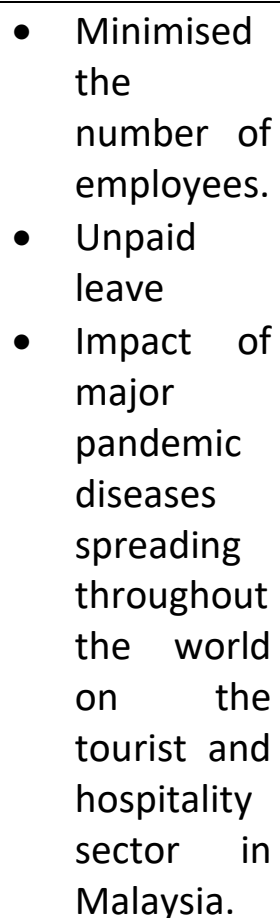 & $\begin{array}{l}\text { - Help the } \\
\text { authority } \\
\text { to } \\
\text { establish } \\
\text { future } \\
\text { precauti } \\
\text { ons and } \\
\text { best } \\
\text { policies. }\end{array}$ & $\begin{array}{l}\text { There are } \\
\text { many } \\
\text { financial } \\
\text { support } \\
\text { given by } \\
\text { the } \\
\text { Governme } \\
\text { nt to the } \\
\text { affected } \\
\text { person in } \\
\text { various } \\
\text { sectors. }\end{array}$ \\
\hline $\begin{array}{l}\text { - James, } \\
\text { Payne, } \\
\text { Gil \& } \\
\text { Mervar } \\
\text { (2021) } \\
\text { - Croatia }\end{array}$ & 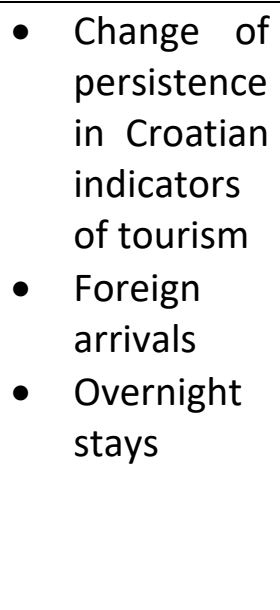 & $\begin{array}{l}\text { Recursi } \\
\text { ve } \\
\text { estimat } \\
\text { ion of a } \\
\text { fraction } \\
\text { al } \\
\text { integra } \\
\text { tion } \\
\text { model. }\end{array}$ & $\begin{array}{l}\text { - Decline of } \\
\text { arrival and } \\
\text { overnight } \\
\text { stays in } \\
\text { the foreign } \\
\text { countries } \\
\text { - Maximise } \\
\text { in the } \\
\text { sustainabili } \\
\text { ty of both } \\
\text { indicators } \\
\text { of tourism. }\end{array}$ & 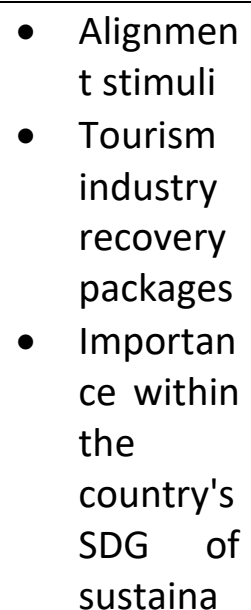 & $\begin{array}{l}\text { Croatia } \\
\text { also } \\
\text { provided } \\
\text { the } \\
\text { financial } \\
\text { support to } \\
\text { the needy } \\
\text { people } \\
\text { with their } \\
\text { recovery } \\
\text { package } \\
\text { program. }\end{array}$ \\
\hline
\end{tabular}




\begin{tabular}{|c|c|c|c|c|c|}
\hline & & & & $\begin{array}{l}\text { ble } \\
\text { tourism. }\end{array}$ & \\
\hline $\begin{array}{l}\text { - Jaipuria, } \\
\text { Parida \& } \\
\text { Ray } \\
\text { (2020) } \\
\text { - India }\end{array}$ & 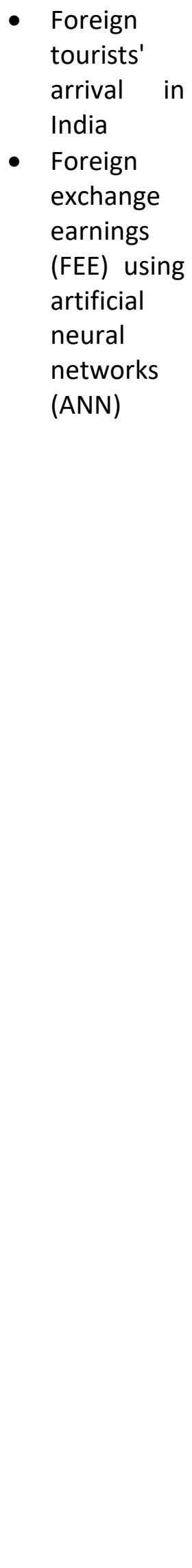 & $\begin{array}{l}\text { - Model } \\
\text { for the } \\
\text { Artificia } \\
\text { I Neural } \\
\text { Networ } \\
k \text { (ANN) } \\
\text { to } \\
\text { forecas } \\
t \quad \text { the } \\
\text { impact } \\
\text { of an } \\
\text { outbre } \\
\text { ak of } \\
\text { covID- } \\
19 \text { The } \\
\text { from } \\
\text { the } \\
\text { arrival } \\
\text { of } \\
\text { interna } \\
\text { tional } \\
\text { visitors } \\
\text { in India }\end{array}$ & $\begin{array}{l}\text { - The } \\
\text { reduction } \\
\text { in the } \\
\text { number of } \\
\text { foreign } \\
\text { visitors } \\
\text { results in } \\
\text { the } \\
\text { reduction } \\
\text { of the FEE. }\end{array}$ & $\begin{array}{l}\text { - The } \\
\text { mutual } \\
\text { interacti } \\
\text { on } \\
\text { between } \\
\text { the } \\
\text { covID- } \\
19 \\
\text { pandemi } \\
\text { c and the } \\
\text { tourist } \\
\text { sector is } \\
\text { widely } \\
\text { understo } \\
\text { od. } \\
\text { Enhance } \\
\text { d } \\
\text { contribut } \\
\text { ion by } \\
\text { forecasti } \\
\text { ng the } \\
\text { number } \\
\text { of } \\
\text { foreign } \\
\text { visitors } \\
\text { and } \\
\text { exchange } \\
\text { rates } \\
\text { (monthly } \\
\text { data) } \\
\text { input } \\
\text { to assist } \\
\text { the } \\
\text { into the } \\
\text { ANN } \\
\text { model is } \\
\text { made by } \\
\text { projectin } \\
\text { g foreign } \\
\text { tourism } \\
\text { arrivals } \\
\text { and the } \\
\text { FEE. }\end{array}$ & $\begin{array}{l}\text { The } \\
\text { models of } \\
\text { the } \\
\text { Artificial } \\
\text { Neural } \\
\text { Network } \\
\text { (ANN) have } \\
\text { been used } \\
\text { to forecast } \\
\text { the } \\
\text { influence } \\
\text { on the } \\
\text { arrival of } \\
\text { foreign } \\
\text { visitors } \\
\text { from India } \\
\text { from the } \\
\text { CoviD-19 } \\
\text { pandemic } \\
\text { that will } \\
\text { enable the } \\
\text { different } \\
\text { players in } \\
\text { the } \\
\text { tourism } \\
\text { sector to } \\
\text { help the } \\
\text { people of } \\
\text { India boost } \\
\text { their } \\
\text { economic } \\
\text { activity. }\end{array}$ \\
\hline
\end{tabular}




\begin{tabular}{|c|c|c|c|c|c|}
\hline & & & & $\begin{array}{l}\text { sector in } \\
\text { recoverin } \\
\mathrm{g} \text { from } \\
\text { the } \\
\text { current } \\
\text { circumst } \\
\text { ance was } \\
\text { recomm } \\
\text { ended } \\
\text { for } \\
\text { various } \\
\text { stakehol } \\
\text { ders in } \\
\text { the } \\
\text { tourist } \\
\text { industry. }\end{array}$ & \\
\hline $\begin{array}{l}\text { - Saha \& } \\
\text { Bhowmi } \\
\text { k (2020) } \\
\text { - South } \\
\text { Asia }\end{array}$ & $\begin{array}{l}\text { - Analysis } \\
\text { between } \\
\text { the nations } \\
\text { of the } \\
\text { socioecono } \\
\text { mic } \\
\text { consequen } \\
\text { ces of } \\
\text { Coronaviru } \\
\text { s (COVID- } \\
\text { 19). }\end{array}$ & $\begin{array}{l}\text { Case } \\
\text { Study } \\
\text { (Secon } \\
\text { dary } \\
\text { data } \\
\text { analysis } \\
\text { ) }\end{array}$ & 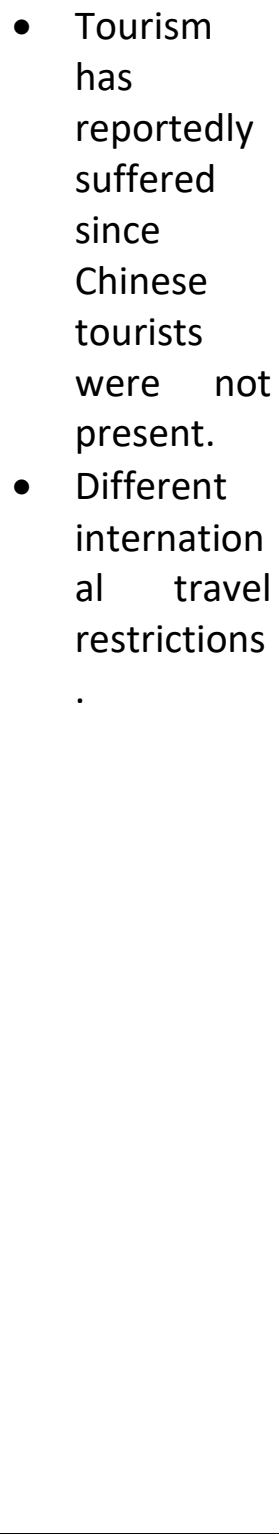 & $\begin{array}{l}\text { The } \\
\text { severe } \\
\text { social } \\
\text { effect of } \\
\text { the } \\
\text { COVID- } \\
19 \text { issue } \\
\text { has been } \\
\text { addresse } \\
\text { d by } \\
\text { restrictin } \\
\text { g mobility } \\
\text { in several } \\
\text { countries } \\
\text { of South- } \\
\text { Asia. }\end{array}$ & $\begin{array}{l}\text { - Signific } \\
\text { ant } \\
\text { regulat } \\
\text { ory } \\
\text { measur } \\
\text { es } \\
\text { encom } \\
\text { pass: } \\
\text { - restrict } \\
\text { ion of } \\
\text { non- } \\
\text { essenti } \\
\text { al } \\
\text { activiti } \\
\text { es } \\
\text { Closure } \\
\text { of } \\
\text { educati } \\
\text { on } \\
\text { institut } \\
\text { ions } \\
\text { Increas } \\
\text { e } \\
\text { awaren } \\
\text { ess of } \\
\text { stay at } \\
\text { home } \\
\text { progra } \\
\text { m. }\end{array}$ \\
\hline
\end{tabular}




\begin{tabular}{|c|c|c|c|c|c|}
\hline & & & & & $\begin{array}{l}\text { - Imposit } \\
\text { ion of } \\
\text { lock- } \\
\text { outs } \\
\text { for } \\
\text { certain } \\
\text { cities } \\
\text { - Unlocki } \\
\text { ng of } \\
\text { particul } \\
\text { ar } \\
\text { operati } \\
\text { ons for } \\
\text { the } \\
\text { normal } \\
\text { circulat } \\
\text { ion of } \\
\text { require } \\
\text { d } \\
\text { goods } \\
\text { on the } \\
\text { market } \\
\text { Work } \\
\text { from } \\
\text { home }\end{array}$ \\
\hline $\begin{array}{l}\text { - Ranasin } \\
\text { ghe, } \\
\text { Damunu } \\
\text { pola, } \\
\text { Wijesun } \\
\text { dara, } \\
\text { Karunar } \\
\text { athna, } \\
\text { Nawarat } \\
\text { hna, } \\
\text { Gamage } \\
\text { Ranawe } \\
\text { era, \& } \\
\text { Idroos } \\
\text { (2020) } \\
\text { Sri Lanka }\end{array}$ & 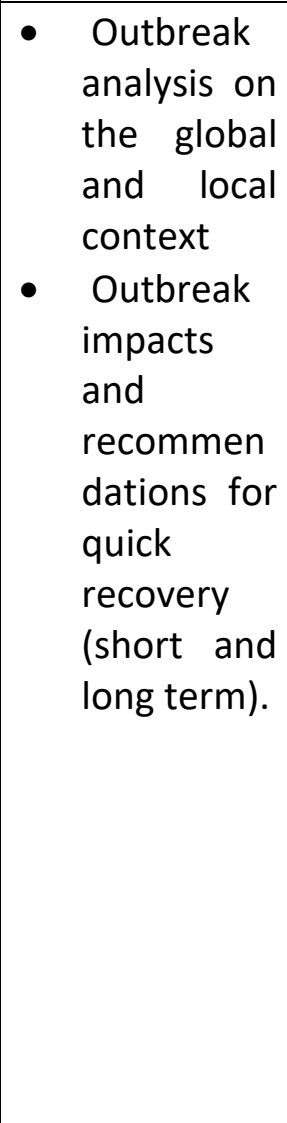 & $\begin{array}{l}\text { System } \\
\text { atic } \\
\text { Article } \\
\text { Review }\end{array}$ & $\begin{array}{l}\text { Major } \\
\text { retail } \\
\text { industry } \\
\text { issues, } \\
\text { food, } \\
\text { consumer } \\
\text { goods and } \\
\text { delivery of } \\
\text { health care }\end{array}$ & $\begin{array}{l}\text { - After the } \\
\text { cOVID } \\
\text { pandemi } \\
\text { c, the } \\
\text { attractiv } \\
\text { eness for } \\
\text { gatherin } \\
\text { gs and } \\
\text { festivals } \\
\text { is much } \\
\text { diminish } \\
\text { ed as } \\
\text { customer } \\
\text { s are } \\
\text { motivate } \\
\text { d by } \\
\text { value } \\
\text { consider } \\
\text { ations } \\
\text { such as } \\
\text { security, } \\
\text { friendshi }\end{array}$ & $\begin{array}{l}\text { The } \\
\text { majority of } \\
\text { tourism } \\
\text { was carried } \\
\text { out in Sri } \\
\text { Lanka and } \\
\text { other } \\
\text { industries } \\
\text { in Sri Lanka } \\
\text { were also } \\
\text { affected. }\end{array}$ \\
\hline
\end{tabular}




\begin{tabular}{|c|c|c|c|c|c|}
\hline & & & & $\begin{array}{l}\mathrm{p} \text { and } \\
\text { pricing. }\end{array}$ & \\
\hline $\begin{array}{ll}\text { - } & \text { Marco } \\
& \text { Camilli } \\
& (2020) \\
\text { - } \quad \text { Italy }\end{array}$ & $\begin{array}{l}\text { - This paper } \\
\text { investigate } \\
\mathrm{s} \text { the } \\
\text { impact in } \\
\text { the tourist } \\
\text { industry of } \\
\text { the COVID- } \\
19 \\
\text { pandemic } \\
\text { outbreak in } \\
\text { Italy } \\
\text { The } \\
\text { present } \\
\text { circumstan } \\
\text { ces, } \\
\text { governmen } \\
\text { t measures } \\
\text { and the } \\
\text { reaction by } \\
\text { the } \\
\text { business } \\
\text { sector in } \\
\text { the } \\
\text { summer of } \\
2020 \text { are } \\
\text { analysed. }\end{array}$ & $\begin{array}{l}\text { - Second } \\
\text { ary } \\
\text { data } \\
\text { analysis }\end{array}$ & $\begin{array}{l}\text { - } \text { Massive } \\
\text { losses in } \\
\text { tourism } \\
\text { and } \\
\text { transport } \\
\text { industry } \\
\text { - In the year } \\
2020-21 \\
\text { the supply } \\
\text { chain might } \\
\text { lose } \\
\text { between } \\
24 \text { billion to } \\
66 \text { billion in } \\
\text { turnover. } \\
\text { Many } \\
\text { tourism } \\
\text { businesses } \\
\text { are unlikely } \\
\text { to survive. }\end{array}$ & $\begin{array}{l}\text { - The } \\
\text { repositio } \\
\text { ning for } \\
\text { the next } \\
\text { two } \\
\text { years of } \\
\text { many } \\
\text { Italian } \\
\text { tour } \\
\text { operator } \\
\text { s at least, } \\
\text { with a } \\
\text { focus on } \\
\text { safety } \\
\text { and } \\
\text { sanitary } \\
\text { requirem } \\
\text { ents, on } \\
\text { an Italian } \\
\text { destinati } \\
\text { on. } \\
\text { Helping } \\
\text { small } \\
\text { enterpris } \\
\text { es to } \\
\text { meet the } \\
\text { challeng } \\
\text { es of } \\
\text { changing } \\
\text { requirem } \\
\text { ents of } \\
\text { visitors } \\
\text { and new } \\
\text { needs of } \\
\text { tourism } \\
\text { firms, } \\
\text { not just } \\
\text { with tax } \\
\text { cuts but } \\
\text { also with } \\
\text { targeted } \\
\text { investme } \\
\text { nts in } \\
\text { sustaina }\end{array}$ & $\begin{array}{l}\text { - Substa } \\
\text { ntial } \\
\text { price } \\
\text { increas } \\
\text { es } \\
\text { - Reduce } \\
\text { availabl } \\
\text { e seats } \\
\text { in } \\
\text { aircraft } \\
\text {, hotels } \\
\text { and } \\
\text { railway } \\
\text { s } \\
\text { Decrea } \\
\text { sing } \\
\text { beach } \\
\text { spaces } \\
\text { Closing } \\
\text { of a } \\
\text { large } \\
\text { numbe } \\
r \\
\text { touris of } \\
\text { m- } \\
\text { related } \\
\text { small } \\
\text { compa } \\
\text { nies. } \\
\text { Curren } \\
\text { tly, the } \\
\text { Italian } \\
\text { Govern } \\
\text { ment is } \\
\text { workin } \\
\text { g on } \\
\text { incenti } \\
\text { ve } \\
\text { progra } \\
\text { ms at } \\
\text { various } \\
\text { levels } \\
\text { in } \\
\text { with }\end{array}$ \\
\hline
\end{tabular}




\begin{tabular}{|c|c|c|c|c|c|}
\hline & & & & $\begin{array}{l}\text { tourism } \\
\text { and a } \\
\text { sustaina } \\
\text { ble } \\
\text { economy } \\
\text {. }\end{array}$ & $\begin{array}{l}\text { certain } \\
\text { payme } \\
\text { nts and } \\
\text { taxes } \\
\text { postpo } \\
\text { ned } \\
\text { until } \\
2020 \text {. } \\
\text { To } \\
\text { invite } \\
\text { people } \\
\text { to } \\
\text { spend } \\
\text { their } \\
\text { summe } \\
r \\
\text { holiday } \\
\text { s in } \\
\text { Italy in } \\
\text { order } \\
\text { to } \\
\text { overco } \\
\text { me the } \\
\text { loss. }\end{array}$ \\
\hline $\begin{array}{l}\text { - Deb \& } \\
\text { Md. Nafi } \\
\text { (2020) } \\
\text { - Banglad } \\
\text { esh }\end{array}$ & $\begin{array}{l}\text { - Analyse the } \\
\text { effect of } \\
\text { the COVID- } \\
19 \\
\text { pandemic } \\
\text { on the } \\
\text { tourism } \\
\text { industry in } \\
\text { Bangladesh } \\
\text {. }\end{array}$ & $\begin{array}{l}\text { - Second } \\
\text { ary } \\
\text { data } \\
\text { analysis } \\
\text {. }\end{array}$ & $\begin{array}{l}\text { - Strict } \\
\text { prohibition } \\
\text { of } \\
\text { accessing } \\
\text { tourist sites } \\
\text { was } \\
\text { introduced } \\
\text { by local } \\
\text { governmen } \\
\text { ts in } \\
\text { Bangladesh } \\
\text { - } \\
\text { Mass } \\
\text { domestic } \\
\text { and } \\
\text { internation } \\
\text { al flights } \\
\text { have been } \\
\text { canceled } \\
\text { and airlines } \\
\text { sector } \\
\text { damages of } \\
\text { USD } 30\end{array}$ & $\begin{array}{l}\text { Many } \\
\text { visitors } \\
\text { abroad } \\
\text { have } \\
\text { canceled } \\
\text { hotel } \\
\text { bookings } \\
\text { - Significa } \\
\text { nt } \\
\text { financial } \\
\text { losses for } \\
\text { the hotel } \\
\text { and } \\
\text { tourist } \\
\text { sector } \\
\text { Global } \\
\text { travel } \\
\text { restrictio } \\
\text { ns } \\
\text { directly } \\
\text { influence } \\
\text { the } \\
\text { airlines }\end{array}$ & $\begin{array}{l}\text { Due to the } \\
\text { hotel } \\
\text { reservatio } \\
\mathrm{n} \\
\text { cancellatio } \\
\mathrm{n} \text {, aircraft } \\
\text { cancellatio } \\
\mathrm{ns} \text { by } \\
\text { tourist } \\
\text { incoming } \\
\text { or } \\
\text { outgoing } \\
\text { and other } \\
\text { issues } \\
\text { relating to } \\
\text { tourism, } \\
\text { many } \\
\text { tourism } \\
\text { staff have } \\
\text { been } \\
\text { affected. }\end{array}$ \\
\hline
\end{tabular}




\begin{tabular}{|c|c|c|c|c|c|}
\hline & & & $\begin{array}{l}\text { million } \\
\text { have been } \\
\text { generated. } \\
\text { Some } \\
\text { efforts } \\
\text { have been } \\
\text { put in place } \\
\text { by } \\
\text { Biman Ban } \\
\text { gladesh } \\
\text { airlines to } \\
\text { decrease } \\
\text { their } \\
\text { losses, } \\
\text { including a } \\
\text { 10\% cut of } \\
\text { basic staff } \\
\text { wages, a } \\
\text { terminatio } \\
\mathrm{n} \text { to the } \\
\text { overtime } \\
\text { pay and a } \\
\text { reduction } \\
\text { of all other } \\
\text { benefits. } \\
\text { All on- } \\
\text { arrival visas } \\
\text { have been } \\
\text { stopped for } \\
\text { visitors } \\
\text { from every } \\
\text { country. }\end{array}$ & $\begin{array}{l}\text { and } \\
\text { cause } \\
\text { tremend } \\
\text { ous } \\
\text { financial } \\
\text { pressure } \\
\text { s. }\end{array}$ & \\
\hline $\begin{array}{l}\text { - Aminath } \\
\text { Shafiya } \\
\text { Adam, } \\
\text { Aminath } \\
\text { Riyaz, } \\
\text { Shazla } \\
\text { Moham } \\
\text { ed, } \\
\text { Raniya } \\
\text { Sobir, } \\
\text { Fathima } \\
\text { th } \\
\text { Nasiha } \\
\text { Abdul } \\
\text { Muhaim }\end{array}$ & $\begin{array}{l}\text { - The resort } \\
\text { employees' } \\
\text { experience } \\
\text { and } \\
\text { worries at } \\
\text { the } \\
\text { outbreak o } \\
\text { f COVID } 19 .\end{array}$ & $\begin{array}{l}\text { - Intervie } \\
\mathrm{w}\end{array}$ & $\begin{array}{l}\text { - The results } \\
\text { show } \\
\text { heightened } \\
\text { employee } \\
\text { concern } \\
\text { over COVID } \\
19 \\
\text { - Possible } \\
\text { health and } \\
\text { safety } \\
\text { impacts } \\
\text { - Economic } \\
\text { impacts on } \\
\text { the resort } \\
\text { workers as }\end{array}$ & 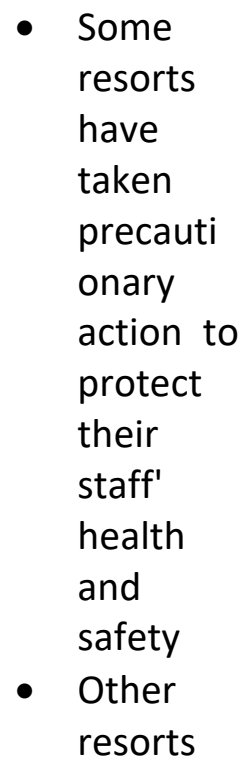 & $\begin{array}{l}\text { Covid19 } \\
\text { has carried } \\
\text { out } \\
\text { tourism in } \\
\text { the } \\
\text { Maldives, } \\
\text { since it is } \\
\text { Maldives' } \\
\text { most } \\
\text { reliable } \\
\text { industry. } \\
\text { Most } \\
\text { employees } \\
\text { are really } \\
\text { concerned }\end{array}$ \\
\hline
\end{tabular}




\begin{tabular}{|c|c|c|c|c|c|}
\hline $\begin{array}{l}\text { in, } \\
\text { Aminath } \\
\text { Sudha, } \\
\text { \& } \\
\text { Fathima } \\
\text { th } \\
\text { Shadiya, } \\
\text { (2020) } \\
\text { - Maldive } \\
\text { s }\end{array}$ & & & $\begin{array}{l}\text { a result of } \\
\text { the resort's } \\
\text { restricted } \\
\text { paid } \\
\text { packages } \\
\text { for the } \\
\text { unexpecte } \\
d \text { closure of } \\
\text { its resorts } \\
\text { due to the } \\
\text { pandemic. }\end{array}$ & $\begin{array}{l}\text { have } \\
\text { been less } \\
\text { bothered }\end{array}$ & $\begin{array}{l}\text { about a } \\
\text { large } \\
\text { number of } \\
\text { safety } \\
\text { concerns. }\end{array}$ \\
\hline $\begin{array}{l}\text { - Ranjit } \\
\text { Sah, } \\
\text { Shailend } \\
\text { ra } \\
\text { Sigdel, } \\
\text { Akihiko } \\
\text { Ozaki, } \\
\text { Yasuhiro } \\
\text { Kotera, } \\
\text { Divya } \\
\text { Bhandar } \\
\text { i, } \\
\text { Priyanka } \\
\text { Regmi, } \\
\text { Ali A. } \\
\text { Rabaan, } \\
\text { Rachana } \\
\text { Mehta, } \\
\text { Mahesh } \\
\text { Adhikari } \\
\text { ' } \\
\text { Namrata } \\
\text { Roy, } \\
\text { Kuldeep } \\
\text { Dhama } \\
\text { \& } \\
\text { Tetsuya } \\
\text { Tanimot } \\
\text { o, } \\
\text { (2020) } \\
\text { Nepal }\end{array}$ & 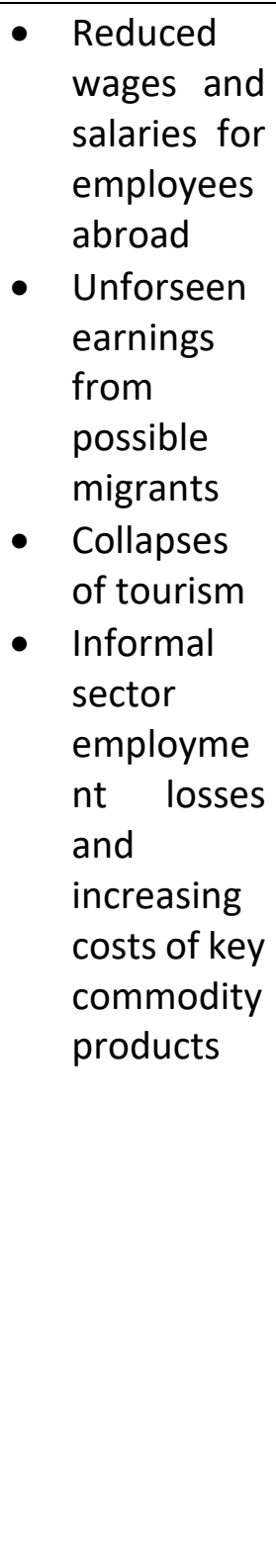 & $\begin{array}{l}\text { - Second } \\
\text { ary } \\
\text { data } \\
\text { analysis }\end{array}$ & $\begin{array}{l}\text { - The fall of } \\
\text { national } \\
\text { and } \\
\text { internation } \\
\text { al tourism } \\
\text { in January } \\
2020 \\
\text { follows a } \\
\text { decrease of } \\
2 \% \\
\text { arrivals in } \\
\text { from } 2019 . \\
\text { More than } \\
10 \\
\text { visitors, } \\
\text { many o00 } \\
\text { whom } \\
\text { were } \\
\text { evicted, } \\
\text { who had } \\
\text { also visited } \\
\text { Nepal } \\
\text { before to } \\
\text { lockdown } \\
\text { were } \\
\text { stranded. }\end{array}$ & 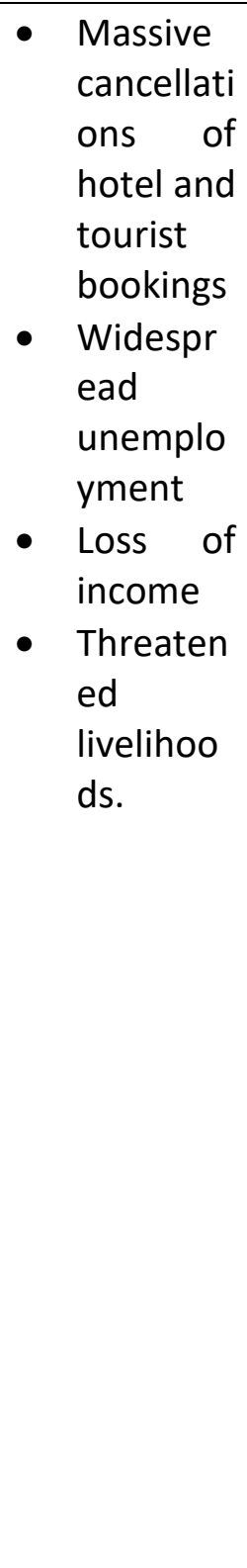 & $\begin{array}{l}\text { The } \\
\text { cancelatio } \\
\text { ns of the } \\
\text { rooms and } \\
\text { other } \\
\text { tourism } \\
\text { activity } \\
\text { affected } \\
\text { excessive } \\
\text { droppings } \\
\text { of visitor } \\
\text { arrivals to } \\
\text { Nepal. This } \\
\text { leads to } \\
\text { unemploy } \\
\text { ment, } \\
\text { income } \\
\text { loss and } \\
\text { many } \\
\text { others. }\end{array}$ \\
\hline $\begin{array}{l}\text { - Binh Do, } \\
\text { Ninh } \\
\text { Nguyen, } \\
\text { Charles } \\
\text { Darwin, } \\
\end{array}$ & $\begin{array}{l}\text { - } \text { COVID-19's } \\
\text { major } \\
\text { influences } \\
\text { are falling } \\
\text { income, }\end{array}$ & $\begin{array}{ll}\text { - } & \text { Intervie } \\
\text { w } \\
\text { - Survey }\end{array}$ & $\begin{array}{l}\text { - The COVID- } \\
19 \\
\text { pandemic } \\
\text { mainly has } \\
\text { lowered }\end{array}$ & $\begin{array}{l}\text { - } \text { Tour } \\
\text { operator } \\
\mathrm{s} \text { in } \\
\text { Vietnam } \\
\text { are alert }\end{array}$ & $\begin{array}{r}\text { Tour } \\
\text { operators } \\
\text { are aware } \\
\text { of the need } \\
\text { to }\end{array}$ \\
\hline
\end{tabular}




\begin{tabular}{|c|c|c|c|c|}
\hline \begin{tabular}{|l} 
Clare \\
D'Souza, \\
Huu Duc \\
Bui \& Thi \\
Nguyen \\
Hong \\
Nguyen \\
(2021) \\
- Vietnam
\end{tabular} & $\begin{array}{l}\text { higher cost } \\
\text { and } \\
\text { redundanci } \\
\text { es for } \\
\text { employees. }\end{array}$ & $\begin{array}{l}\text { revenues, } \\
\text { higher } \\
\text { expenses } \\
\text { and the } \\
\text { redundanc } \\
y \text { of } \\
\text { employees. }\end{array}$ & $\begin{array}{l}\text { of the } \\
\text { need for } \\
\text { adjustme } \\
\text { nt and } \\
\text { anticipat } \\
\text { e } \\
\text { governm } \\
\text { ent } \\
\text { financial } \\
\text { help to } \\
\text { resolve } \\
\text { the problem. }\end{array}$ & $\begin{array}{l}\text { restructure } \\
\text { and they } \\
\text { anticipate } \\
\text { governme } \\
\text { nt financial } \\
\text { assistance } \\
\text { to } \\
\text { overcome } \\
\text { the crisis } \\
\text { and focus } \\
\text { more on } \\
\text { short- and } \\
\text { medium- } \\
\text { term (i.e. } \\
\text { restriction, } \\
\text { perseveran } \\
\text { ce and } \\
\text { plans than } \\
\text { on long- } \\
\text { term (i.e. } \\
\text { innovative) } \\
\text { planning in } \\
\text { response } \\
\text { to coviD- } \\
\text { anding }\end{array}$ \\
\hline
\end{tabular}

\section{Summary}

The past ten articles on COVID-19 and the tourist sector are summarised in Table 1. The primary emphasis of the researchers in this overview is the influence of COVID-19 on the worldwide tourism sector. In a word, most of the nations said that they were greatly affected, notably in tourism, such as hospitality, airlines and other industries. Furthermore, several nations established financial assistance programmes, which would help the countries that had lost their jobs, leaving unpaid or wage cuts. In Malaysia, this may be observed when the Malaysian Government launched the program of financial support and other initiatives. The majority of the firm has limited the number of staff and unpaid leave to remain in the sector. The Croatia authorities and the Government have created a political effort that could include aligning the incentive and recovery packages for the tourism industry to decrease the number of foreign arrivals and stays over the night and to increase the level of persistence in both tourism indicators. At the same time, India said that reducing the number of international tourists leads to lower foreign exchange income (FEE).

An added contribution is the forecast of international tourist arrivals and the FEE, as an input to the ANN model, using the number of foreign tourists and the monthly exchange rates. Such as in South Asia, the severe social effects of the Covid-19 crisis were overcome by the limitation imposed on people's movements in several nations in South Asia. After the Covid19 pandemic, Sri Lanka attracted events and festivals rapidly since customers are motivated by the value aspects, such as safety, friendship and pricing. Meanwhile, many Italian tour 
operators have repositioned themselves at least for the next two years on the Italian destination, emphasising safety and sanitary requirements. Moreover, with the reduction in taxation and targeted investment in sustainable tourism and sustainable economies, the Government and the Authority have helped small businesses, driven by innovative approaches and focusing on the new demands of tourism companies and unique needs. In Bangladesh, numerous outside tourists have cancelled hotel reservations and caused substantial financial losses to hotel and tourism sectors.

Bangladesh's worldwide travel restrictions also directly affect airlines and cause tremendous economic strain. In addition, Maldives is a country that relies on the tourist sector for the most part in its income. It highlights the heightened concern and influence on the health and safety of employees about exposure to Covid-19, moreover, because of the administration's limited wage packages to cope with the unexpected close of resorts. Due to the pandemic, the economic impact on resort employees. In addition, several huge hotel and tourist reservation cancellations are being made, with widespread unemployment, a loss of revenue and endangered livelihoods in Nepal. Lastly, Vietnam's tour operators are aware of the need for restructuring in Vietnam directly in the tourist sector and expect the Government to get financial help to overcome the problem.

\section{Discussion}

Pandemic Covid-19 certainly have a tremendous influence on tourism and the economy of the most critical industries for most countries. Undoubtedly, tourism is a significant industry in the global economy. The tourism sector in 2019 accounted for $29 \%$ of the world's service exports and over 300 million jobs. It is an essential source of income and employment for industrialised and developing countries. The worldwide drop in tourist numbers may have disastrous economic repercussions, as many developing nations are primarily dependent on tourism. According to the United Nations Trade and Development Conference, tourism accounts for over half of the GDP of several nations, including numerous small developing island countries (SIDS) (2021).

As shown in Table 1, most countries have said that their people face job losses, pay reduction, and unpaid leaves. Most countries have the same problems, and their economies as an individual and national income in their whole have indirectly been affected. The partial or complete lockdown in much of the country made it difficult for individuals. The most influential persons lost the job because of the pandemic, particularly the one in the tourism industry. The study and implementation of various regulations will help the sector as the industry transitions during the recovery stages. First, the policy will be crucial from international border opening cooperation to defining common criteria and assisting employees in significantly broadening test availability. The industry should also explore regulations that encourage travel, facilitate securing and improve skills while rethinking the site of work, among others, as it adjusts to the following normal situation. As the industry improves its sustainability, governments may increase environmental protection and interact closely with local communities and the business sector, creating and executing a new workforce perspective. Ultimately, improved coordination between Government and Government and alignment between the public and private sectors will be crucial to accomplish recovery 
Table 2: Tourism Recovery Recommendation Plan Based On The Reviewed Articled

Tourism Recovery Recommendation Plan Based On The Reviewed Articled

\begin{tabular}{|c|c|}
\hline $\begin{array}{l}\text { Major impacted } \\
\text { effect }\end{array}$ & Proposed recovery plan \\
\hline $\begin{array}{l}\text { - Unemployment } \\
\text { - Getting Jobless } \\
\text { - } \text { Cut Wages } \\
\text { - Unpaid Leaves } \\
\text { - Hotel Room } \\
\text { - Cancellation } \\
\text { - Flight Seat } \\
\text { - Sancellation } \\
\text { - Stranded Tourist } \\
\text { Deficiency Traffic }\end{array}$ & 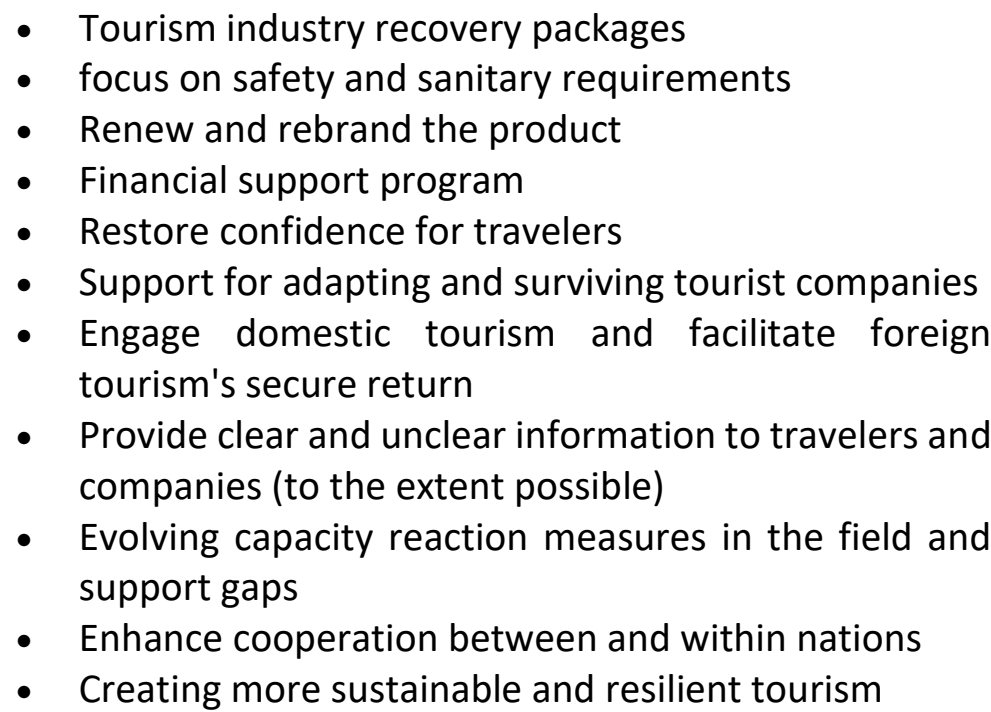 \\
\hline
\end{tabular}

(Quratul and Yuliandi, 2021)

Most of the tourism activities have resumed and contributed to reducing the impact on employment and companies in some places. But only when foreign tourism returns would make recovery possible. To securely eliminate travel restrictions, global cooperation and evidence-based solutions are necessary. According to OECD (2020), they have made some countermeasures to help boost the tourism industry, especially restoring confidence for travelers by ensuring the SOPs are conducting tightly. The place's staff must be in their pink of health. Besides that, it also mentioned having support for adapting and surviving tourist companies as many countries have implemented. In addition, the OECD (2020) also stated that to engage domestic tourism and facilitate foreign tourism's safe return for the new key policy recommend from them.

Moreover, to provide clear and reduce as much as possible the unclear information to travellers and companies. This approach will help all parties to understand and take precautions in this time. Next, another key policies from OECD (2020) is evolving capacity reaction measures in the field and support gaps and enhance cooperation between and within nations. Lastly, from OECD (2020), the new key policy is to create more sustainable and resilient tourism to build recovery for the tourism sector. Although adaptable policy approaches are required to allow the tourist sector to live with the virus in the short to medium term, it is necessary to look beyond it and adopt measures to learn from the crisis that has highlighted weaknesses in governments' readiness and reaction capability industries. There is a need for coordinated action by all governments and the private sector at all levels. The crisis represents a chance for the future to replenish tourism. Tourism is at a crossroads, and tomorrow's tourism will be shaped by the measures implemented today. Governments must consider the longer-term consequences of this crisis and capitalise on digitalisation, 
support a low carbon transition and promote the structural transformation necessary for building a more potent, more stable and more sustainable tourism industry OECD (2020).

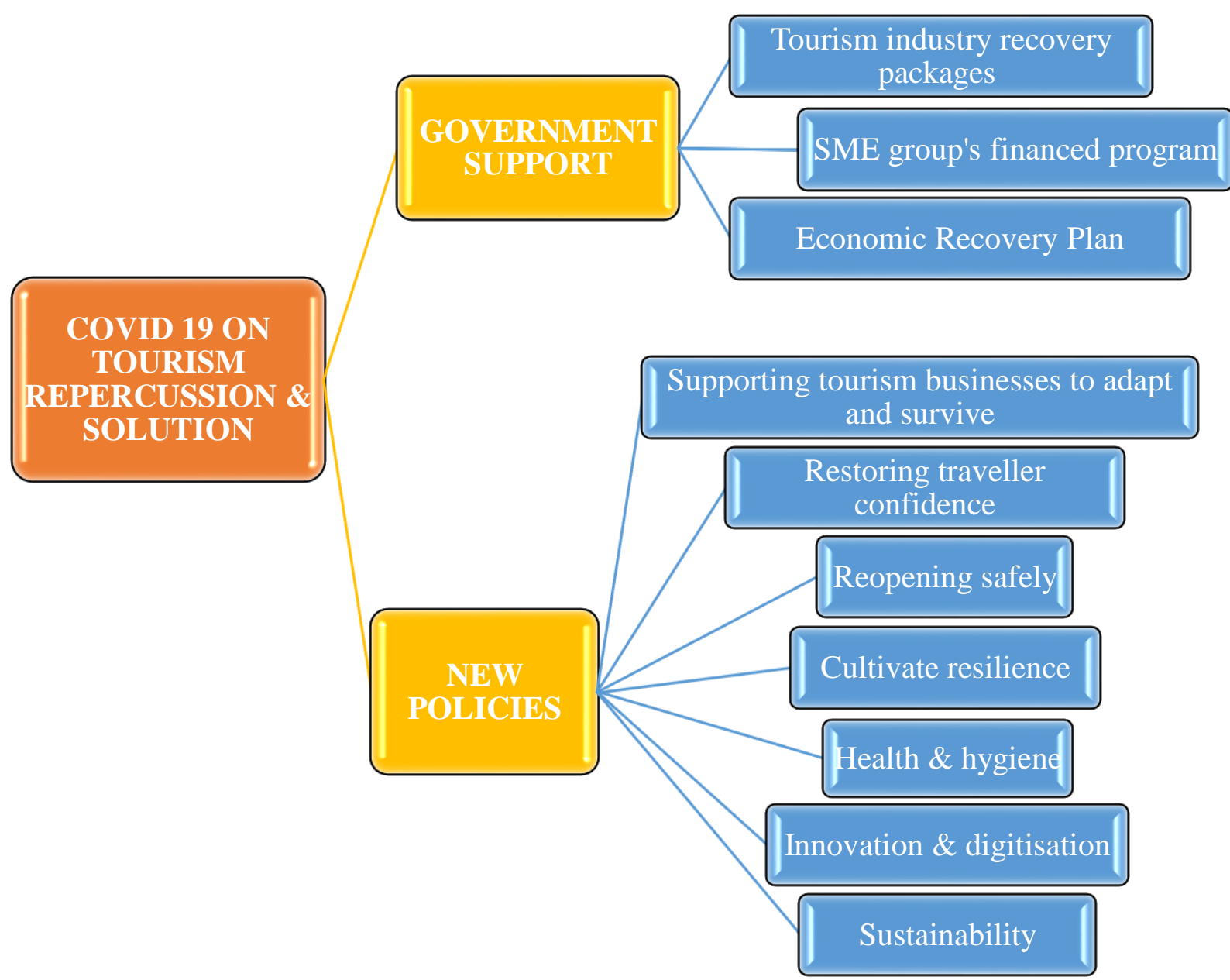

Figure 3: Illustration of Covid 19 On Tourism Repercussion \& Solution

(Quratul and Yuliandi, 2021)

Significant losses and disruptions due to COVID-19 continue to occur across the world. The travel \& tourism business is not least shocked by these failures. Since $90 \%$ of the world's population acclimated to travel limits and others stayed home in dread of the virus itself, it almost stopped. The weight of zero income on big and small communities, dependent on tourism, and millions of people were flooded or disposed of in a couple of short months. Over 121 million jobs worldwide in tourism may lose to COVID-19, which was based on the WTTC baseline scenario. It is estimated at US\$ 3,4 trillion of global GDP; this figure, a downside scenario by the time we do not have the international coordination and leadership, could be as high as 197 million before the end of this year. However, the travel \& tourism private sector leaders in the face of hardship have stayed firm. Over the past few months, the industry has tried to improve passenger safety, supply its employees, and assist local communities. Indeed, industry leaders are taking advantage of the crisis to help the sector develop inclusively and sustainably. While government support has been expanded, there is still a greater need to coordinate and implement policies that promote the revival of the industry. A worldwide, 
coordinated strategy will be required, the present seamless travel experience will be enhanced, new technologies integrated will be combined and global health and hygiene regulations will finally re-establish travellers' confidence.

Throughout this current frontier, health and safety are crucial. The short and medium-term behaviour of the customer is guided by personal experiences, professional advice and distance considerations. Businesses will have to work even closer with their extended value chains to guarantee that similar protocols, such as WTTC's Safe Travels standard, are ready and implemented. Trust, widespread communications and reliable information flow will, in this context, be a major driver for retrieval in the industry among travellers and employees, companies and suppliers, visitors and local communities. In the drive for innovation and integration of new technology, COVID-19 is proving an unanticipated catalyst for the travel \& Tourism sector. Digital acceptance and consumption are growing with residual orders, and customers increasingly demand contactless technology, including biometrics, as an essential need for a secure and smooth travelling experience. Cybersecurity is increasingly vital only when remote work becomes a rule in the near and medium term and identities are digitised. Although digitisation provides enormous scope, measures are required to ensure that staff and community groups aren't abandoned apart. The globe has been revitalised to address social, environmental and institutional sustainability from broad unemployment and antiracism campaigns to restoring natural habitats and their influence on ecosystems. Species have increased public awareness and support for the conservation of wildlife as well as for the sustainability of the ocean. Based on their environmental record and support of diversity and integration, businesses have been subject to increased monitoring.

In comparison to others, travel \& tourism has a unique capacity to protect and involve vulnerable populations while decreasing poverty and inequality due to its strong engagement by women, minorities and the young. This is the moment to speed up the significant improvements for the future generations that will have a long-lasting effect. Companies and destinations need substantial help from governments to convert existing barriers into recovery possibilities in today's socioeconomic situation. Government actions to mitigate COVID 19's impact on the travel and tourism sector, including reducing travel restrictions, supporting liquidity and fiscal measures, protecting workers and introducing private sector health protocols, promoting tourism and investing, and fostering innovation, have already been taken been undertaken by governments.

In Malaysia, various income-based financial support programs are classified under the B40, M40 and T20 categories for all Malaysians. The SME group's financed program allocatted with RM 3000, the national incentive program on youth up to RM 15000 and the automatic 6month moratorium held in 2020 and continuing in 2021 are also additional financial assistance program. Contrary, a loan program COVID-19 has been established in Croatia to safeguard jobs, and tax cuts are also granted to the Government. Throughout many levels of society in the country, both the Authority and the Italian government work on the incentive programs. Vietnamese tour operators are conscious of the need to restructure and anticipate financial help from the Government to overcome the crisis and guarantee low-interest loans, delayed tax payments, insurance premiums. Vietnamese tour operators hope to get financial support.

Finally, like in Indonesia, the Economic Recovery Plan is prepared and Australia's government support. Therefore, most nations struggle to increase their tourism and economy, and the state works to aid the country. Most of the Government's financial support helps the majority of those in difficulty living in this epidemic age. There are several financial assistance and 
encouragement programs in Malaysia, such as Bantuan Sara Hidup, Bantuan Prihatin Rakyat, and Bakul Prihatin Negara, to equally aid Malaysian individuals and families. In addition, Malaysia announced reducing in the Overnight Policy Rate (OPR). The decision to reduce the OPR is to stimulate investors in the market to enhance the movement of liquid money and rekindle the country's economic growth. This incentive will surely attract many people or business people to loan to the bank because of the low-interest rate. However, the tour operator is still having some issues since he has taken the loan to improve the business, but because of the pandemic, they have to cease operating and have a debt to be paid for without a flow of money.

\section{Conclusion and Recommendation}

Today, the severe public health crisis has become a catastrophic global disaster for the tourist and business sectors. The rehabilitation must proceed hand-in-hand with the recovery of other sectors, the battle against the epidemic and the economic recovery. The proposed measures should thus be applied progressively in response to developments. For stakeholders in the hotel business, for example, tourists and staff's safety has become a key issue. As a result, this paper highlights tourism and the world's economies, which have been catastrophically affected by the pandemic COVID-19, since the activities of aircraft all over the globe have ceased. Furthermore, the hotel industry is struggling to comply with MCO. The majority loses jobs, unemployment, unpaid leaves and the world economy in the worst possible conditions due to this Covid 19 epidemic. The paper highlighted the appearance and impact of the virus on tourism and commercial enterprises. As the recommendation, the whole country impacted by the Covid-19 epidemic may assist people by providing all government people with financial support. In addition, low-tax OPRs announced by the Government to individuals could attract many people to get loans at economic rates, the funds it can spend and indirectly assist in strengthening the country's economies. Finally, to stimulate the economy, the Government can promote local products like shopping in the nation and urge people to support it as indirectly supporting the country's economies.

\section{References}

Alehegn, M. M. (2021). The Economic Impact of COVID-19 in Ethiopia, 2(22), 83-90.

Barrafrem, K. (2020). Financial well-being, COVID-19, and the financial better-, (May). https://doi.org/10.31234/osf.io/tkuaf

Camilli, M. (2020). The Impact of COVID-19 on Italian Tourism:, (May), 15-19. https://doi.org/10.13140/RG.2.2.17541.58087

Deb, S. K., \& Nafi, S. (n.d.). Impact of COVID-19 Pandemic on Tourism : Perceptions from, (December 2019).

Franco-bedoya, S. (2020). Examining the Economic Impact of COVID-19 in India through Daily Electricity Consumption and Nighttime Light Intensity, (June).

Jaipuria, S., Parida, R., \& Ray, P. (2021). The impact of COVID-19 on tourism sector in India. https://doi.org/10.1080/02508281.2020.1846971

No Title. (n.d.), 164(May 2020), 113-126.

Overview, P., \& America, L. (2021). the poorer regions, such as the country's North and, (August 2020), 18-21.

Pak, A., Adegboye, O. A., Adekunle, A. I., \& Rahman, K. M. (2020). Economic Consequences of the COVID-19 Outbreak: the Need for Epidemic Preparedness, 8(May), 1-4. https://doi.org/10.3389/fpubh.2020.00241 
Payne, J. E., Gil-alana, L. A., \& Mervar, A. (2021). Persistence in Croatian tourism : The impact of COVID-19, 1-7. https://doi.org/10.1177/1354816621999969

Ranasinghe, R., Wijesundara, W., Karunarathne, C., \& Nawarathna, D. (2020). TOURISM AFTER CORONA: IMPACTS OF COVID 19 PANDEMIC AND WAY TOURISM AFTER CORONA : IMPACTS OF COVID 19 PANDEMIC AND WAY FORWARD FOR TOURISM, HOTEL AND MICE, (April). https://doi.org/10.13140/RG.2.2.27955.17442

Riyaz, A., Mohamed, S., \& Shadiya, F. (2020). Experiences and concerns during the COVID-19 pandemic : A qualitative research with employees in the tourism sector of the Maldives Experiences and concerns during the COVID - 19 pandemic : A qualitative research with employees in the tourism sector of the, (September).

Roy, S. (2020). ECONOMIC IMPACT OF COVID-19 PANDEMIC ECONOMIC IMPACT OF COVID19 PANDEMIC, (July).

Rpa, A. (n.d.). The Movement Control Order ( MCO ) for COVID-19 Crisis and its Impact on Tourism and Hospitality Sector in Malaysia.

Sah, R., Sigdel, S., Ozaki, A., Kotera, Y., Bhandari, D., Regmi, P., ... Glasg, F. R. (2020). Impact of COVID-19 on tourism in Nepal, (July), 1-3. https://doi.org/10.1093/jtm/taaa105

Saha, N. K., \& Bhowmik, S. C. (2020). Socioeconomic Impact of Coronavirus ( COVID-19) Pandemic in South Asia, 11(6), 22-28. https://doi.org/10.9790/5933-1106042228

Tran, B. X., Nguyen, H. T., Le, H. T., \& Latkin, C. A. (2020). Impact of COVID-19 on Economic Well-Being and Quality of Life of the Vietnamese During the National Social Distancing, (September). https://doi.org/10.3389/fpsyg.2020.565153 